\title{
Recent results in kaon physics
}

\author{
Zuzana Kucerova*t \\ Comenius University Bratislava \\ E-mail: zuzana.kucerova@cern.ch
}

A review of the present experimental status of the $K \rightarrow \pi v \bar{v}\left(K_{\pi v v}\right)$ and other kaon decay analyses at experiments NA62 (CERN) and KOTO (J-PARC) is given. The $K_{\pi v v}$ decay is one of the best candidates among the rare meson decays for indirect searches for new physics in the mass ranges complementary to those accessible by current accelerators. The Standard Model (SM) prediction of the branching fraction $(\mathcal{B})$ of the $K_{\pi v v}$ decay is lower than $10^{-10}$ in both neutral and charged modes. The NA62 experiment aims to measure the $\mathcal{B}$ of the charged mode with better than $10 \%$ precision. Three candidate events, compatible with the SM prediction, have been observed from a sample of $2.12 \times 10^{12} K^{+}$decays collected in 2016 and 2017 by NA62. More than twice the statistics is available in the 2018 dataset currently being analysed. The KOTO experiment in Japan aims to measure $\mathcal{B}\left(K_{L} \rightarrow \pi^{0} v \bar{v}\right)$ using a technique similar to NA62, but with much lower momentum. In the first dataset taken in 2015 zero signal candidate events were observed. The current status of the analysis of the 2016-2018 dataset with 1.4 times more data is presented. Finally, the most recent results of other physics analyses at the NA62 experiment are summarised.

18th International Conference on B-Physics at Frontier Machines - Beauty2019 -

29 September $/ 4$ October, 2019

Ljubljana, Slovenia

* Speaker.

${ }^{\dagger}$ for the NA62 Collaboration: R. Aliberti, F. Ambrosino, R. Ammendola, B. Angelucci, A. Antonelli, G. Anzivino, R. Arcidiacono, T. Bache, M. Barbanera, J. Bernhard, A. Biagioni, L. Bician, C. Biino, A. Bizzeti, T. Blazek, B. BlochDevaux, V. Bonaiuto, M. Boretto, M. Bragadireanu, D. Britton, F. Brizioli, M.B. Brunetti, D. Bryman, F. Bucci, T. Capussela, J. Carmignani, A. Ceccucci, P. Cenci, V. Cerny, C. Cerri, B. Checcucci, A. Conovaloff, P. Cooper, E. Cortina Gil, M. Corvino, F. Costantini, A. Cotta Ramusino, D. Coward, G. D’Agostini, J. Dainton, P. Dalpiaz, H. Danielsson, N. De Simone, D. Di Filippo, L. Di Lella, N. Doble, B. Dobrich, F. Duval, V. Duk, J. Engelfried, T. Enik, N. Estrada-Tristan, V. Falaleev, R. Fantechi, V. Fascianelli, L. Federici, S. Fedotov, A. Filippi, M. Fiorini, J. Fry, J. Fu, A. Fucci, L. Fulton, E. Gamberini, L. Gatignon, G. Georgiev, S. Ghinescu, A. Gianoli, M. Giorgi, S. Giudici, F. Gonnella, E. Goudzovski, C. Graham, R. Guida, E. Gushchin, F. Hahn, H. Heath, E.B. Holzer, T. Husek, O. Hutanu, D. Hutchcroft, L. Iacobuzio, E. Iacopini, E. Imbergamo, B. Jenninger, J. Jerhot, R.W. Jones, K. Kampf, V. Kekelidze, S. Kholodenko, G. Khoriauli, A. Khotyantsev, A. Kleimenova, A. Korotkova, M. Koval, V. Kozhuharov, Z. Kucerova, Y. Kudenko, J. Kunze, V. Kurochka, V. Kurshetsov, G. Lanfranchi, G. Lamanna, E. Lari, G. Latino, P. Laycock, C. Lazzeroni, M. Lenti, G. Lehmann Miotto, E. Leonardi, P. Lichard, L. Litov, R. Lollini, D. Lomidze, A. Lonardo, P. Lubrano, M. Lupi, N. Lurkin, D. Madigozhin, I. Mannelli, G. Mannocchi, A. Mapelli, F. Marchetto, R. Marchevski, S. Martellotti, P. Massarotti, K. Massri, E. Maurice, M. Medvedeva, A. Mefodev, E. Menichetti, E. Migliore, E. Minucci, M. Mirra, M. Misheva, N. Molokanova, M. Moulson, S. Movchan, M. Napolitano, I. Neri, F. Newson, A. Norton, M. Noy, T. Numao, V. Obraztsov, A. Ostankov, S. Padolski, R. Page, V. Palladino, A. Parenti, C. Parkinson, E. Pedreschi, M. Pepe, M. PerrinTerrin, L. Peruzzo, P. Petrov, Y. Petrov, F. Petrucci, R. Piandani, M. Piccini, J. Pinzino, I. Polenkevich, L. Pontisso, Yu. Potrebenikov, D. Protopopescu, M. Raggi, A. Romano, P. Rubin, G. Ruggiero, V. Ryjov, A. Salamon, C. Santoni, G. Saracino, F. Sargeni, S. Schuchmann, V. Semenov, A. Sergi, A. Shaikhiev, S. Shkarovskiy, D. Soldi, V. Sugonyaev, M. Sozzi, T. Spadaro, F. Spinella, A. Sturgess, J. Swallow, S. Trilov, P. Valente, B. Velghe, S. Venditti, P. Vicini, R. Volpe, M. Vormstein, H. Wahl, R. Wanke, B. Wrona, O. Yushchenko, M. Zamkovsky, A. Zinchenko. 


\section{Introduction}

The decay $K \rightarrow \pi v \bar{v}[1,2]$ is a Flavour Changing Neutral Current (FCNC) loop process strongly suppressed in the Standard Model (SM) by a quadratic GIM mechanism [3] and CKM matrix elements $[4,5]$. Short distance contributions dominate the decay amplitude. Hadronic matrix elements extracted from well measured kaon decays allow for high precision of the decay rate calculation within the SM. The current predictions for the branching fractions in the SM are [6]

$$
\mathcal{B}\left(K^{+} \rightarrow \pi^{+} v \bar{v}\right)=(8.4 \pm 1.0) \times 10^{-11} \quad \mathcal{B}\left(K_{L} \rightarrow \pi^{0} v \bar{v}\right)=(3.4 \pm 0.6) \times 10^{-11}
$$

with the uncertainties being dominated by the CKM matrix elements extracted from the tree-level processes. The $K_{\pi v v}$ decays not only play an important role in testing of the SM properties, as they provide crucial contributions to a fit of the Unitarity triangle, but they are also sensitive to the physics beyond the SM. Many extensions of the SM predict significant deviations in branching fractions of these decays $[7,8,9,10,11,12]$ due to new particles in the loops of $K_{\pi v v}$ diagrams.

Branching fractions of both charged and neutral modes of $K_{\pi v v}$ decay have been measured previously at Brookhaven National Laboratory (BNL). The combined measurement by the E787 and E949 collaborations with a kaon decay-at-rest technique resulted in $\mathcal{B}\left(K^{+} \rightarrow \pi^{+} v \bar{v}\right)=\left(17.3_{-10.5}^{+11.5}\right) \times$ $10^{-11}$ [13], which is consistent with the SM expectation within the statistical uncertainty. The NA62 collaboration observed one $K^{+} \rightarrow \pi^{+} v \bar{v}$ candidate event in the 2016 dataset [14]. The current best upper limit for the branching fraction of the neutral mode has been set by the E391a collaboration at $90 \% \mathrm{CL}: \mathcal{B}\left(K_{L} \rightarrow \pi^{0} v \bar{v}\right)<2.6 \times 10^{-8}$ [15]. The KOTO experiment observed zero signal candidate events in the 2015 dataset [16].

\section{The NA62 Experiment at CERN}

The NA62 experiment for rare charged kaon decays is a part of the kaon physics program at CERN offering an approach to probing new physics through precision measurements complementary to the high energy frontier [17]. It has a broad physics program covering precision measurements of rare kaon decays, searches for lepton flavour (LFV) and lepton number violating (LNV) decays forbidden in the SM, as well as searches for exotic particles connecting the SM with a hidden sector. The primary goal of the NA62 experiment is the measurement of $\mathcal{B}\left(K^{+} \rightarrow \pi^{+} v \bar{v}\right)$ with less than $10 \%$ relative uncertainty. For this purpose a novel technique of kaon decays-in-flight is exploited.

NA62 started taking physics data in 2016 and continued until November 2018. Results presented in this article were obtained from 2016 and 2017 datasets corresponding to $1.1 \times 10^{11}$ and $2 \times 10^{12}$ kaon decays, respectively.

\subsection{The beam and detector}

NA62 is a fixed target experiment connected to the CERN SPS by a proton beam line. The primary proton beam, with momentum of $400 \mathrm{GeV} / c$ from the SPS, is directed on a Be target, producing a secondary positive beam. The secondary beam, with a total particle rate of about $750 \mathrm{MHz}$ and the mean momentum of $75 \mathrm{GeV} / c$ consists mainly of pions and protons with a $6 \%$ contribution of kaons. A schematic layout of the NA62 experiment is shown in the Fig.1. 


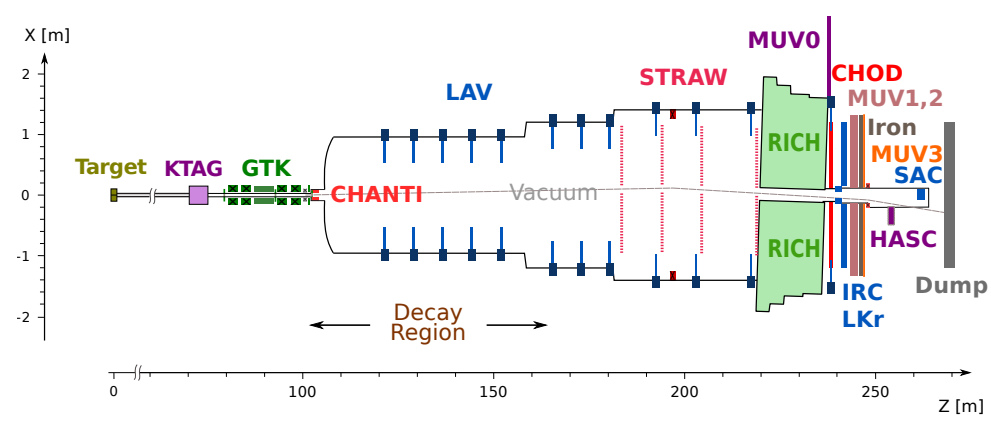

Figure 1: Schematic layout of the NA62 Experiment in the $x z$ plane.

A secondary beam line navigates the beam through a set of collimators and achromats towards the upstream section of the NA62 detector, which is dedicated to measurement of the beam particle properties. A differential Cherenkov counter (KTAG) is used for time measurement of the kaon component of the beam with better than 70 ps precision. A beam spectrometer (GTK) is a silicon pixel detector consisting of three identical stations and several sets of dipole magnets. It provides the momentum and direction measurement of each beam particle. All GTK stations have to sustain the beam intensity of about $450 \mathrm{MHz}$. Finally, an upstream counter (CHANTI) is placed directly after the last GTK station to veto events in which an interaction of a beam particle with material of the GTK station occured. The upstream section is followed by a large evacuated cylindrical tank in which a $60 \mathrm{~m}$ long fiducial decay volume is defined, where about $6 \%$ of the beam kaons decay. The decay region is followed by downstream detectors designed for the detection and measurement of the properties of the kaon decay products. Four chambers of the downstream spectrometer (STRAW) together with a dipole magnet between the middle two chambers provide the momentum and direction measurement of all charged decay products. Several sets of photon detectors (LAV, SAC, IRC, LKr) are spread along the decay region and downstream section of the NA62 detector and they hermetically cover angles up to $50 \mathrm{mrad}$ from the beam axis. The electromagnetic calorimeter $(\mathrm{LKr})$ is used as a part of the photon veto system and participates in the particle identification (PID). Two charged hodoscopes (CHODs) placed after the STRAW are used for time measurement of the charged particles. A ring imaging Cherenkov detector (RICH) is a key element in PID and provides clear separation of pions, positrons and muons in the momentum range of 15-35 GeV/c. The muon veto system consists of two hadronic calorimeters (MUV1, MUV2) designed to distinguish between muons and pions, and a plastic scintillator tile detector (MUV3) placed at the end of the NA62 detector behind an $80 \mathrm{~cm}$ thick iron wall. The veto system is complemented by another hadronic calorimeter (HASC) and a scintillator hodoscope (MUV0) providing further rejection of specific cases of hadronic kaon decays. Further details about the NA62 beam and detector can be found in [18].

\subsection{The NA62 $K^{+} \rightarrow \pi^{+} v \bar{v}$ selection and analysis}

The kinematic variable squared missing mass reflects the signature of a $K^{+} \rightarrow \pi^{+} v \bar{v}$ decay with one positive pion in the final state and a missing energy in the form of neutrinos. It is defined as $m_{m i s s}^{2}=\left(P_{K^{+}}-P_{\pi^{+}}\right)^{2}$, where $P_{K^{+}}$is the measured kaon four-momentum and $P_{\pi^{+}}$is the measured four-momentum of the charged decay product in the $m_{\pi^{+}}$hypothesis. Expressing the branching 
fractions of the $K^{+} \rightarrow \pi^{+} v \bar{v}$ and the most probable kaon decays as a function of $m_{\text {miss }}^{2}$ suggests the definition of two separate signal regions used for the $\mathcal{B}\left(K^{+} \rightarrow \pi^{+} v \bar{v}\right)$ measurement. Region 1 is placed between the $K^{+} \rightarrow \mu^{+} v\left(K_{\mu 2}\right)$ and $K^{+} \rightarrow \pi^{+} \pi^{0}\left(K_{2 \pi}\right)$ peaks and region 2 between $K_{2 \pi}$ and $K^{+} \rightarrow \pi^{+} \pi^{+} \pi^{-}\left(K_{3 \pi}\right)$ peaks. As shown in Fig.2, the signal regions are chosen such to minimize the contribution from gaussian tails of these background distributions as well as from other kaon decays. However, non-gaussian and radiative tails still stretch across the two signal regions. The estimated contributions from the tails of the $K_{\mu 2}, K_{2 \pi}$ and $K_{3 \pi}$ decays in the signal regions are validated in several control regions defined around the peaks of the distributions. Both control and signal regions are kept blinded until the full selection is finalised.

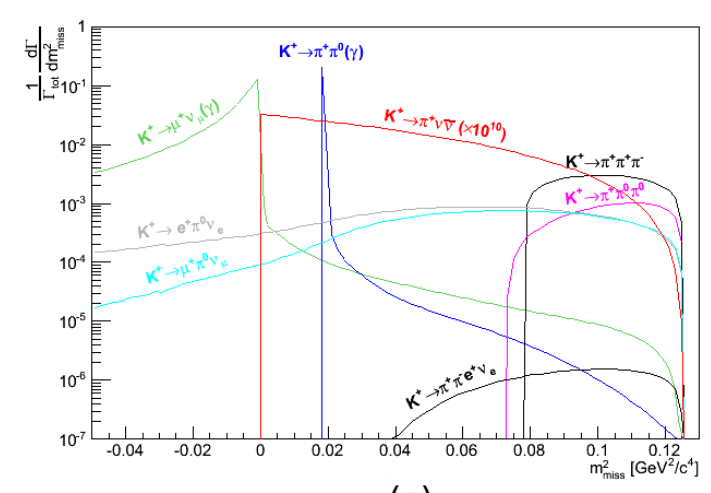

(a)

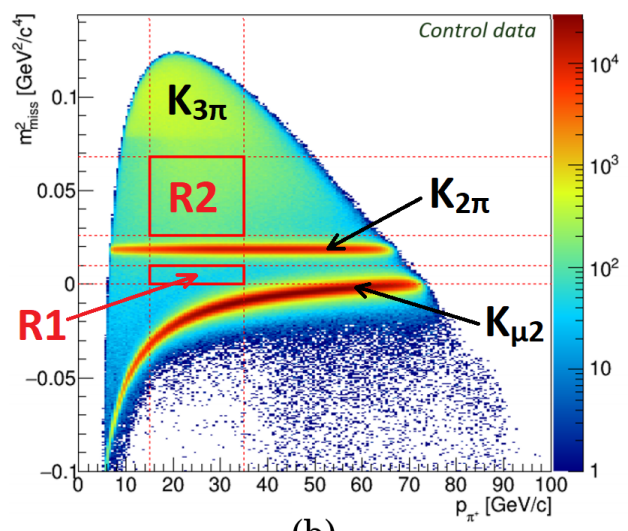

(b)

Figure 2: (a): Theoretical $m_{\text {miss }}^{2}$ distribution for signal and main $K^{+}$decay modes assuming the $\pi^{+}$mass of the charged track; the signal (red) is multiplied by a factor of $10^{10}$ [18]. (b): $m_{\text {miss }}^{2}$ distribution as a function of track momentum for events selected with Control Trigger stream. The signal regions R1 and R2 are drawn as red boxes for reference. Contributions of the three main kaon decays are identified.

Two independent trigger streams are used to collect the data. A dedicated trigger stream (Physics Trigger) is used for selection of the $K^{+} \rightarrow \pi^{+} v \bar{v}$ candidate events, while a minimum-bias trigger stream (Control Trigger) is used for the normalization, efficiency and selection performance studies. A low-level trigger (L0) makes decisions about data readout from detectors based on logical signals produced by the RICH, CHODs, LKr, LAV and MUV3 (in case of the Control Trigger only one of the CHODs is used and a downscaling factor 400 is applied). After positive L0 verdict data from KTAG, LAVs and STRAW are processed by the software trigger (L1) to make more advanced decision using higher level information obtained by applying simplified reconstruction algorithms similar to those used offline.

Signal selection is based on several key pillars: kinematics, particle identification and veto. In the first step $\mathrm{K}^{+}$decays inside the decay region with single track topology are selected. For this purpose information from upstream detectors KTAG and GTK is combined with that from downstream spectrometer STRAW to match a beam kaon with a downstream track. The selection proceeds by a $\pi^{+}$identification using the RICH detector and combined information from the calorimeters (LKr, MUV1, MUV2) and MUV3. Events with any extra in-time energy deposition in the calorimeters not corresponding to the $\pi^{+}$track are rejected. Multitrack events with one or more tracks not reconstructed by the STRAW spectrometer but detected by LKr, CHODs or HASC are also rejected. Photon veto detectors LAV, SAV, IRC are used to suppress events with photons 
interacting with the material before reaching the LKr. A $\mu^{+}$and $\pi^{0}$ suppression of the order of $10^{8}$ as well as more than $64 \% \pi^{+}$identification efficiency are achieved. To protect against misreconstruction of the event kinematics or mismatch between the $\pi^{+}$track and kaon, a final cut on the $m_{m i s s}^{2}$ is performed in a space of three squared missing mass variables, combining momentum of the downstream track measured either by the STRAW or by the RICH and momentum of the kaon as measured by the GTK or the nominal beam momentum.

Two categories of background events are identified - events coming from other kaon decays and upstream background events coming from early kaon decays or inelastic interactions inside the achromat of the GTK detector. In the former case, the fraction of $K_{\mu 2}, K_{2 \pi}$ and $K_{3 \pi}$ events entering the signal regions due to kinematic tails is determined on data with Control triggered samples. The kinematic factors measured to be of the order of $10^{-4}$ are used to estimate the residual background contribution in the signal regions after the $K^{+} \rightarrow \pi^{+} v \bar{v}$ selection. The background contribution coming from other kaon decays is estimated using Monte Carlo samples. The upstream background turns out to be the most prominent one in the 2017 data analysis. In case of an early kaon decay or interaction with the material in the beam achromat before the final collimator and the last GTK station, the pion track could pass through the hole of the final collimator, enter the decay region and reach the downstream detectors. Additionally, a scattering could occur in the first STRAW station resulting in a change of momentum of the pion track, which is then measured by the STRAW. If an in-time beam particle is present, a kaon momentum could be measured by the GTK and a fake vertex could be reconstructed inside the decay region. The position of the pion track extrapolated back to the plane of the final collimator (before the last GTK station) indicates the mechanism of production of an upstream background event. A strong cut suppressing this background is imposed, thus lowering the signal acceptance significantly. The residual upstream background contribution is estimated from both data and simulation.

\subsection{Results}

The measured single event sensitivity (SES) of the $K^{+} \rightarrow \pi v \bar{v}$ analysis of the 2017 dataset is $\mathrm{SES}=(3.89 \pm 0.21) \times 10^{-11}$. The dominant contribution to the systematic uncertainty comes from the L0 trigger performance. After the full $K^{+} \rightarrow \pi^{+} v \bar{v}$ selection, the total signal acceptance is $1.34 \%$, which includes the random veto, trigger efficiency and total detector efficiency.

The expected numbers of signal and background events in the two signal regions combined are given in Table 1. The kinematic tails of the $K_{2 \pi}$ decay dominate the background coming from kaon decays. However, the most prominent component is the upstream background, which is a direct result of the increase in the beam intensity from $40 \%$ in 2016 to $55 \%$ of the nominal intensity in 2017.

After unblinding the signal regions two $K^{+} \rightarrow \pi^{+} v \bar{v}$ candidate events are observed in the signal region 2, as shown in Fig. 3. When combined with the results from the 2016 dataset [14], three candidate events have been observed in total while expecting $1.65 \pm 0.31$ background events with total SES $=(0.346 \pm 0.017) \times 10^{-10}$.

Such observation leads to an upper limit on the branching fraction $\mathcal{B}\left(K^{+} \rightarrow \pi^{+} v \bar{v}\right)$ obtained with the $\mathrm{CL}_{\mathrm{s}}$ method [19]:

$$
\mathcal{B}\left(K^{+} \rightarrow \pi^{+} v \bar{v}\right)<2.44 \times 10^{-10} \quad \text { at } 95 \% \mathrm{CL} .
$$




\begin{tabular}{ll}
\hline Process & Expected events in R1+R2 \\
\hline \hline$K^{+} \rightarrow \pi^{+} v \bar{v}(\mathrm{SM})$ & $2.16 \pm 0.12_{\text {stat }} \pm 0.26_{\text {ext }}$ \\
\hline$K^{+} \rightarrow \pi^{+} \pi^{0}(\gamma)(\mathrm{IB})$ & $0.29 \pm 0.03_{\text {stat }} \pm 0.03_{\text {syst }}$ \\
$K^{+} \rightarrow \mu^{+} v_{\mu}(\gamma)(\mathrm{IB})$ & $0.11 \pm 0.02_{\text {stat }} \pm 0.03_{\text {syst }}$ \\
$K^{+} \rightarrow \pi^{+} \pi^{-} e^{+} v_{e}$ & $0.12 \pm 0.05_{\text {stat }} \pm 0.03_{\text {syst }}$ \\
$K^{+} \rightarrow \mu^{+} v_{\mu}\left(\mu^{+} \rightarrow e^{+}\right.$decay) $)$ & $0.04 \pm 0.02_{\text {syst }}$ \\
$K^{+} \rightarrow \pi^{+} \pi^{-} \pi^{+}$ & $0.02 \pm 0.01_{\text {syst }}$ \\
$K^{+} \rightarrow \pi^{+} \gamma \gamma$ & $0.005 \pm 0.005_{\text {syst }}$ \\
Upstream background & $0.9 \pm 0.2_{\text {stat }} \pm 0.2_{\text {syst }}$ \\
\hline \hline Total background & $1.5 \pm 0.2_{\text {stat }} \pm 0.2_{\text {syst }}$ \\
\hline
\end{tabular}

Table 1: Expected numbers of signal and background events in R1 and R2 after applying the $K^{+} \rightarrow \pi^{+} v \bar{v}$ selection on the 2017 dataset.

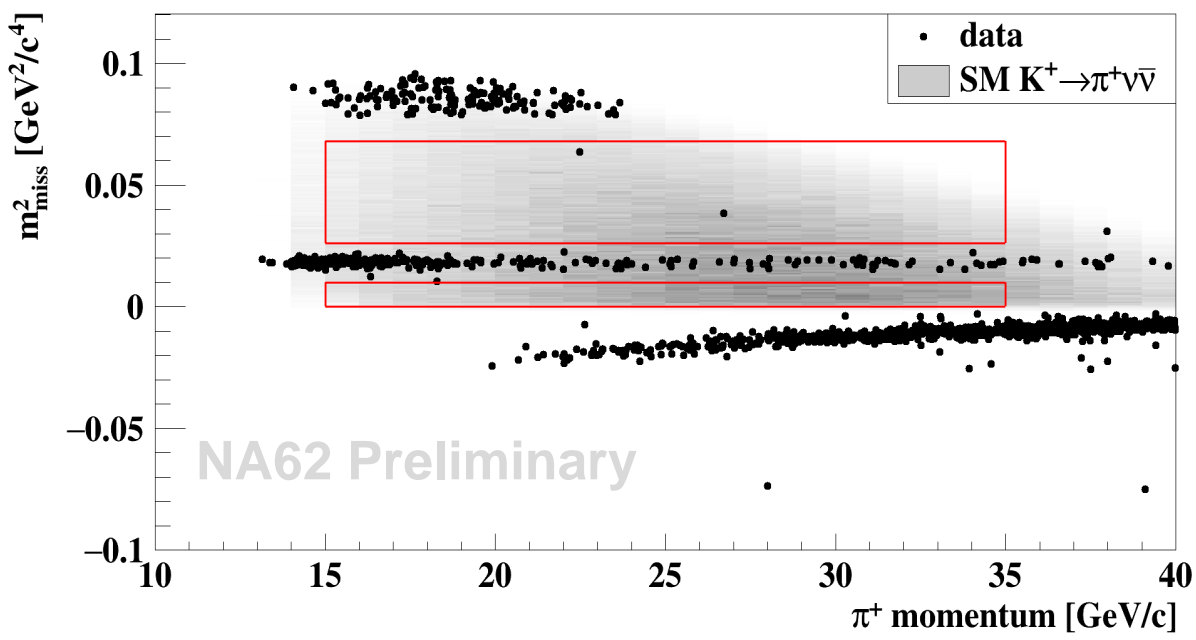

Figure 3: Two $K^{+} \rightarrow \pi^{+} v \bar{v}$ candidate events observed in the signal region 2 after the full signal selection in the 2017 dataset.

\subsection{Other physics analyses at the NA62}

The NA62 experiment offers a unique opportunity to search for physics beyond the SM (BSM) both directly and indirectly in the rare, forbidden and exotic decays of kaons [2] and pions, thus covering wide range of proposed BSM models. A brief summary of several other physics analyses performed at the NA62 experiment in addition to $K_{\pi v v}$ analysis is reported.

\subsection{1 $\pi^{0} \rightarrow$ invisible}

As a natural by-product of the $K^{+} \rightarrow \pi^{+} v \bar{v}$ search, thanks to the rejection power of more than $\mathrm{O}\left(10^{8}\right)$ of any visible $\pi^{0}$ decay, the NA62 is able to perform a high sensitivity search for $\pi^{0}$ decays to invisible particles. In the 2016 and 2017 datasets a large sample of tagged $\pi^{0}$ is available after selecting events with one $\pi^{+}$particle from a $K^{+}$decay in the final state and invariant missing mass compatible with $m_{\pi^{0}}$. Selection of $\pi^{0} \rightarrow$ invisible events relies on the efficiency of the photon veto system ( $\mathrm{LKr}, \mathrm{LAV}, \mathrm{IRC}, \mathrm{SAC}$ ). The background is dominated by $\pi^{0} \rightarrow \gamma \gamma$ events 
with undetected photons, the expected contribution of which is determined from a combination of MC and data-based studies. Region 2 of $K^{+} \rightarrow \pi^{+} v \bar{v}$ analysis with $\pi^{+}$momentum range between $20 \mathrm{GeV} / c$ and $40 \mathrm{GeV} / c$ has been selected as signal region for $\pi^{0} \rightarrow$ invisible search and has been kept blinded. Using the $\mathrm{CL}_{\mathrm{s}}$ method, an upper limit on the branching fraction is set to $\mathcal{B}\left(\pi^{0} \rightarrow\right.$ invisible $)<4.4 \times 10^{-9}$ at $90 \%$ CL from 12 events observed in the signal region.

\subsubsection{Forbidden decays at the NA62}

Many scenarios of BSM physics allow for lepton number violating (LNV) processes forbidden within the SM. Two LNV kaon decays: $K^{+} \rightarrow \pi^{-} e^{+} e^{+}$and $K^{+} \rightarrow \pi^{-} \mu^{+} \mu^{+}$, violating the lepton number conservation by two units and available in some models in the presence of a massive Majorana neutrino U [20], are studied at NA62. A blind analysis is performed in two signal regions of the invariant mass spectrum. The total estimated background in the signal regions is evaluated to be $0.16 \pm 0.03$ for the electron mode and $0.9 \pm 0.41$ for the muon mode. In the 2017 dataset no event is observed in the signal region of the electron mode, while one event is observed in the signal region of the muon mode, as shown in Fig.4. These results translate into improved upper limits on the branching fractions: $\mathcal{B}\left(K^{+} \rightarrow \pi^{-} e^{+} e^{+}\right)<2.2 \times 10^{-10}$ and $\mathcal{B}\left(K^{+} \rightarrow \pi^{-} \mu^{+} \mu^{+}\right)<$ $4.2 \times 10^{-11}$ at $90 \%$ CL [21]. Forseen analysis of the full 2016-2018 dataset is expected to lead to further improvement by a factor of three.
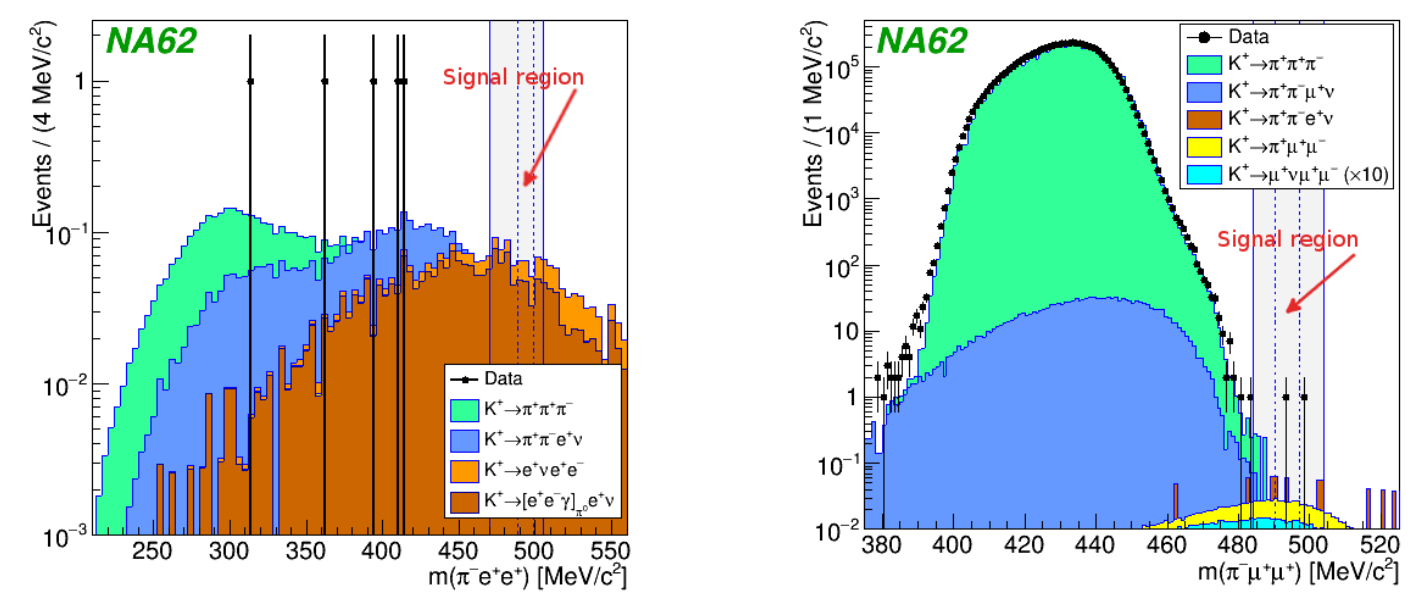

Figure 4: Reconstructed invariant mass of electron (left) and muon (right) mode of $K^{+} \rightarrow \pi^{-} l^{+} l^{+}$decay. Background estimates are overlayed with data. Shaded intervals correspond to signal and control regions of the measurement and were kept blinded during the analysis [21].

\subsubsection{Hidden sector searches: Heavy Neutral Leptons (HNL)}

An example of a BSM model including heavy neutral leptons (HNL) is the neutrino minimal SM [22, 23], which involves adding three massive neutrinos mixing with the SM massless neutrinos to explain dark matter and baryon asymmetry in the Universe. Leptonic $K^{+}$decay channels involving production of $\mathrm{HNL}$ with masses up to $500 \mathrm{MeV} / \mathrm{c}^{2}$ are studied at NA62. The search for $K^{+} \rightarrow l^{+} N(l=e, \mu)$ decays is based on HNL mass scans of missing mass spectra in ranges 136$456 \mathrm{MeV} / c$ and 230-374 MeV/c in positron and muon modes, respectively. No HNL production signal event candidates are observed in the part of the 2017 dataset used for this analysis, and new 
upper limits on the mixing parameter $\mathrm{U}_{l 4}$ in each $\mathrm{HNL}$ mass hypothesis are computed. As shown in Fig.5a, this result improves the previous limits obtained with the 2015 dataset [24] by two (one) orders of magnitude for $\mathrm{U}_{e 4}\left(\mathrm{U}_{\mu 4}\right)$ case.

\subsubsection{Hidden sector searches: Dark Photon $A^{\prime}$}

One of the proposed models of BSM physics introduces a new massive vector field $A^{\prime}$ called a dark photon, which provides vector connection with a hidden sector by mixing with the SM photon with coupling $\varepsilon$ dependent on the mass of $A^{\prime}[25,26]$. NA62 is suited to search for the $A^{\prime}$ in the production channel $\pi^{0} \rightarrow A^{\prime} \gamma$, which is dominant for $M_{A^{\prime}}<M_{\pi^{0}}$. The search relies on tagging $\pi^{0}$ from $K^{+} \rightarrow \pi^{+} \pi^{0}$ decay using $K^{+}$and $\pi^{+} 4$-momentum and imposing a cut on the missing mass. Signal selection requires only one photon in the final state. A dominant background comes from $\pi^{0} \rightarrow \gamma \gamma$ decay with one of the photons undetected. A mass scan over this background is performed, looking for an excess of events at the mass of the dark photon. No statistically significant excess is observed in the subsample of the 2016 dataset [27]. A new upper limit is put on the coupling parameter in the mass region available to NA62, as is shown in the Fig.5b.

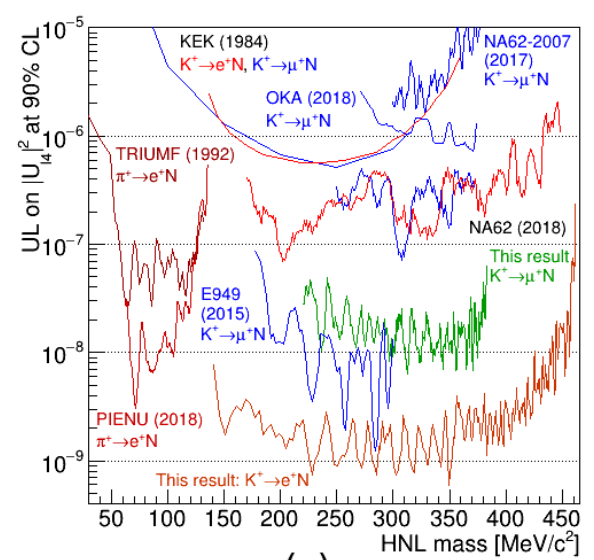

(a)

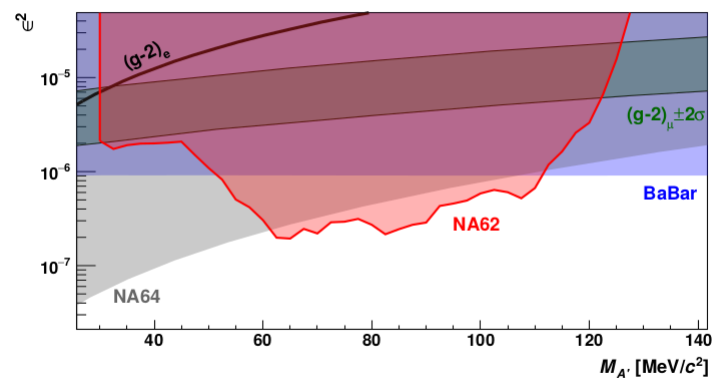

(b)

Figure 5: (a): New upper limits at 90\% CL from NA62 on $\left|\mathrm{U}_{l 4}\right|^{2}$ obtained for each assumed HNL mass (labelled "This result") compared to the limits from earlier HNL production searches. (b): New upper limit at $90 \% \mathrm{CL}$ from NA62 (red region) [27] in the $\varepsilon^{2}$ vs $M_{A^{\prime}}$ plane with $A^{\prime}$ decaying into invisible final states compared to previous limits obtained by NA64 (grey) and BaBar (blue) experiments.

\subsection{Future prospects}

The $K_{\pi^{+} v \bar{v}}^{+}$analysis of the 2018 dataset is currently ongoing. Two times more data than in the 2017 dataset is available and an increased signal acceptance and improved signal-to-background ratio is expected, thanks to a new collimator installed in 2018.

To suppress the upstream background, plans to modify the NA62 beam line setup as well as to add new veto detectors in the beam line for detection of extra particles have been made. A new GTK station will be added to the beam spectrometer to reduce the $K^{+}-\pi^{+}$mistagging probability. After data taking resumes in 2021, NA62 plans to run at $100 \%$ of nominal intensity. This requires improvement in the TDAQ efficiency and good control of the random veto, maintaining it at the same level as in 2018. 
NA62 also plans to collect a large sample of data while operating in a dump mode before the long shutdown at CERN (LS3). In this case the beam intensity can surpass the nominal one by $20-50 \%$ since the dump mode does not reach the performance limits of the detector and the TDAQ system. This data will be used for exotic searches and studies of decays of exotic particles.

A feasibility study for running at considerably higher intensity has been initiated at NA62 having three physics goals in mind, all with similar hardware and readout upgrade requirements. In the proposed Kaon Facility, the precision of the $\mathcal{B}\left(K^{+} \rightarrow \pi^{+} v \bar{v}\right)$ measurement would be improved while running at 4 times the nominal intensity of NA62. A measurement of $\mathcal{B}\left(K_{L} \rightarrow \pi^{0} v \bar{v}\right)$ would be performed as proposed by the KLEVER collaboration [28], complementing the KOTO experiment at J-PARC, as well as a new measurement of $\mathcal{B}\left(K_{L} \rightarrow \pi^{0} e^{+} e^{-}\right)$.

\section{The KOTO Experiment at J-PARC}

J-PARC laboratory in Japan houses several experiments and a high intensity proton accelerator complex. KOTO experiment $[29,30]$ is part of the Hadron Beam facility where a $30 \mathrm{GeV} / c$ proton beam is used to create secondary beams of hadrons by impinging on a $66 \mathrm{~mm}$ thick gold target. A neutral beam is produced at an angle of 16 degrees with respect to the proton beam axis. The beam is composed of neutrons, photons and $K_{L}$ mesons with a peak momentum of $1.4 \mathrm{GeV} / c$. A neutral beam line guides the beam towards a $3 \mathrm{~m}$ long decay volume in vacuum. The decay volume is surrounded by hermetic veto counters and a CSI calorimeter at the far end used for the detection of two photons from the $\pi^{0}$ decay. The veto counters for photons and charged particles ensure that no other particles were produced in the decay and are used for detection of the photons from early $K_{L}$ decays.

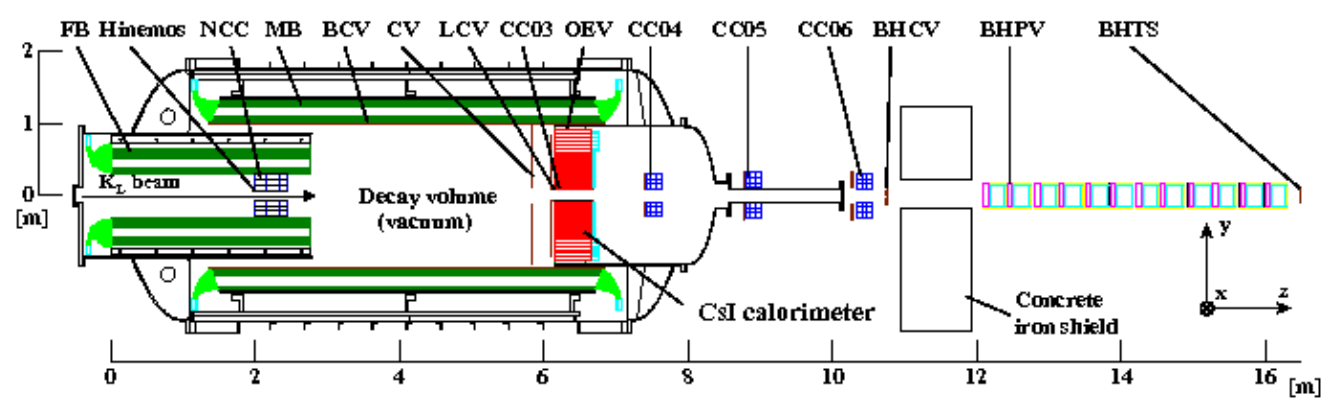

Figure 6: Schematic layout of the KOTO detector [30].

\subsection{The KOTO $K_{L} \rightarrow \pi^{0} v \bar{v}$ selection and analysis}

The signature of the decay $K_{L} \rightarrow \pi^{0} v \bar{v}$ is given by two photons with high $p_{T}$, originating in the decay of $\pi^{0}$, and missing energy in the form of neutrinos. The photons are detected in the CSI calorimeter and a $\pi^{0}$ decay vertex, and transverse momentum is reconstructed assuming the decay occurred on the beam axis. A blinded signal region for $K_{L} \rightarrow \pi^{0} v \bar{v}$ decay selection is defined by the $p_{T}\left(\pi^{0}\right)$ and $Z_{v t x}$ position. The background comes either from other $K_{L}$ decays or from beam halo neutrons interacting with the detector material. 
The analysis of the data sample collected in 2016-2018 by the KOTO experiment follows in the footsteps of the previous analysis performed on the 2015 dataset [16]. After major improvements of the TDAQ, reconstruction and analysis techniques in 2016 and 2017, the signal acceptance increased by a factor of $\sim 1.4$. A SES $=6.9 \times 10^{-10}$ and good control over the background is achieved, with an expected $0.05 \pm 0.02$ background events in the signal region.

\subsection{Results and future prospects}

After unblinding the signal region four events are observed. A first study of these events suggests that one of the background sources might have been underestimated. A careful investigation of the candidate events and reevaluation of all the background sources is currently ongoing.

As presented at the KAON 2019 conference $^{1}$, the measurement of the $\mathcal{B}\left(K_{L} \rightarrow \pi^{0} v \bar{v}\right)$ by the KOTO experiment with precision comparable to the SM prediction requires SES the order of $10^{-11}$. This requires either long running time or major upgrades, not only on the side of KOTO experiment, but also within the accelerator complex of J-PARC. A new power supply for the Main Ring proton accelerator, boosting the beam power towards $100 \mathrm{~kW}$, is planned to be installed in 2020. To further improve the measurement efficiency, a new stage of the experiment, KOTO Step2 [31], is proposed, which includes an increased kaon flux from the neutral beam produced at a smaller production angle. To increase the number of $K_{L}$ decays detected at KOTO, a longer decay region and a larger detector is proposed. Other plans and considerations have been discussed at the KAON 2019 conference.

\section{Conclusions}

The NA62 experiment observed three $K^{+} \rightarrow \pi^{+} v \bar{v}$ candidate events in the 2016 and 2017 datasets. This result not only proved the relevance of the novel decay-in-flight technique and showed that the SM sensitivity can be reached at the NA62, but also established a steady ground for a future $K^{+} \rightarrow \pi^{+} v \bar{v}$ measurement at NA62. Two times more data is already available in the 2018 dataset. Improved signal acceptance and signal-to-background ratio is expected. Major improvements over previous results have also been achieved in other physics analyses at NA62 with 2016 and 2017 datasets. The KOTO experiment observed zero $K_{L} \rightarrow \pi^{0} v \bar{v}$ candidate events in the 2015 dataset. Improved data acquisition and analysis techniques have been used for the collection and analysis of the 2016-2018 dataset. This resulted in only one order of magnitude difference in sensitivity with respect to the SM sensitivity, which is expected to be reached after extensive detector upgrades in 2021. Final results of the full 2016-2018 dataset are expected soon.

\section{Acknowledgments}

This contribution to the proceedings of Beauty2019 conference was supported by Comenius University Bratislava, Slovakia via grant UK/106/2019.

\footnotetext{
${ }^{1}$ KAON 2019 conference website: https : //indico. cern.ch/event/769729/
} 


\section{References}

[1] L. S. Littenberg, CP-violating decay $K_{L}^{0} \rightarrow \pi^{0} v \bar{v}$, Phys. Rev. D 39 (1989) 3322

[2] V. Cirigliano, G. Ecker, H. Neufeld, A. Pich, and J. Portoles, Kaon decays in the standard model, Rev. Mod. Phys. 84 (2012) 399 [hep-ph/1107.6001]

[3] S. Glashow, J. Iliopoulos and L. Maiani, Weak interactions with lepton-hadron symmetry, Phys. Rev. D 2 (1970) 1285

[4] N. Cabibbo, Unitary symmetry and leptonic decays, Phys. Rev. Lett. 10 (1963) 531

[5] M. Kobayashi and T. Maskawa, CP-Violation in the renormalizable theory of weak interactions, Prog. Theor. Phys. 49 (1973) 652

[6] A. J. Buras, D. Buttazzo, J. Girrbach-Noe and R. Knegjens, $K^{+} \rightarrow \pi^{+} v \bar{v}$ and $K_{L} \rightarrow \pi^{0} v \bar{v}$ in the Standard Model: status and perspectives, JHEP 11 (2015) 033 [hep-ph/1503.02693]

[7] S. Fajfer, N. Kosnik and L. Vale Silva, Footprints of leptoquarks: from $R_{K^{(\star)}}$ to $K \rightarrow \pi \nu \bar{v}$, Eur. Phys. J. C 78 (2018) 275 [hep-ph/1802 .00786]

[8] T. Blazek and P. Matak, Left-left squark mixing in $K^{+} \rightarrow \pi^{+} v \bar{v}$ and minimal SUSY, Int. J. of Mod. Phys. A 29 (2014) 1450162 [hep-ph/1410.0055]

[9] M. Blanke, A. J. Buras, B. Duling, K. Gemmler and S. Gori, Rare K and B Decays in a Warped Extra Dimension with Custodial Protection, JHEP 0903 (2009) 108 [hep-ph / 0812 . 38 03]

[10] A. J. Buras, D. Buttazzo and R. Knegjens, $K \rightarrow \pi v \bar{v}$ and $\varepsilon^{\prime} / \varepsilon$ in Simplified New Physics Models, JHEP 1511 (2015) 166 [hep-ph/1507.08672]

[11] M. Blanke, A. J. Buras and S. Recksiegel, Quark flavour observables in the Littlest Higgs model with T-parity after LHC Run 1, Eur. Phys. J. C 78 (2016) 182 [hep-ph/1507.06316]

[12] M. Bordone, D. Buttazzo, G. Isidori and J. Monnard, Probing lepton-flavour universality with $K \rightarrow \pi v \bar{v}$ decays, Eur. Phys. J. 77 (2017) 618

[13] A. V. Artamonov et al., Study of the decay $K^{+} \rightarrow \pi^{+} v \bar{v}$ in the momentum region $140<P_{\pi}<199$ MeV/c, Phys. Rev. D 79 (2009) 092004 [hep-ex/ 0903.0030 ]

[14] E. Cortina Gil et al., First search for $K^{+} \rightarrow \pi^{+} v \bar{v}$ using the decay-in-flight technique, Phys. Lett. B 791 (2019) 156 [hep-ex/1811.08508]

[15] M. Tanabashi et al. (Particle Data Group), Phys. Rev. D 98 (2018) 030001

[16] J. K. Ahn et al. (KOTO collaboration), Search for $K_{L} \rightarrow \pi^{0} v \bar{v}$ and $K_{L} \rightarrow \pi^{0} X^{0}$ Decays at the J-PARC KOTO Experiment, Phys. Rev. Lett 122 (2019) 021802 [hep-ex/1810.09655]

[17] A. Ceccucci et al., Proposal to Measure the Rare Decay $K^{+} \rightarrow \pi^{+} v \bar{v}$ at the CERN SPS, CERN-SPSC-2005-013

[18] E. Cortina Gil et al., The beam and detector of the NA62 experiment at CERN, JINST 12 (2017) P05025 [physics.ins-det/1703.08501]

[19] A. L. Read, Presentation of search results: the $C L_{S}$ technique, J. Phys. G 28 (2002) 2693

[20] A. Atre, T. Han, S. Pascoli and B. Zhang, The Search for Heavy Majorana Neutrinos, JHEP 0905 (2009) 030 [hep-ph/0901.3589]

[21] E. Cortina Gil et al., Search for lepton number violating $K^{+}$decays, Phys. Lett. B 797 (2019) 134794 
[22] T. Asaka and M. Shaposhnikov, The vMSM, Dark Matter and Baryon Asymmetry of the Universe, Phys. Lett. B 620 (2005) 17 [hep-ph/ 0505013 ]

[23] R. Shrock, General theory of weak processes involving neutrinos. I., Phys. Rev. D 24 (1981) 1232

[24] E. Cortina Gil et al., Search for heavy neutral lepton production in $K^{+}$decays, Phys. Lett. B 778 (2018) 137 [hep-ex/1712.00297]

[25] L. B. Okun, Limits of electrodynamics: paraphotons?, Sov. Phys. JETP 56 (1982) 502

[26] B. Holdom, Two U(1)'s and Epsilon Charge Shifts, Phys. Lett. B 166 (1986) 196

[27] E. Cortina Gil et al., Search for production of an invisible dark photon in $\pi^{0}$ decays, JHEP 05 (2019) $182[\mathrm{hep}-\mathrm{ex} / 1903.08767]$

[28] F. Ambrosino et al., KLEVER: An experiment to measure $B R\left(K_{L} \rightarrow \pi^{0} v \bar{v}\right)$ at the CERN SPS, KLEVER-PUB-18-02 [hep-ex/1901.03099]

[29] T. Yamanaka et al., The J-PARC KOTO experiment, PTEP 2012 (2012) $02 \mathrm{~B} 006$

[30] T. Masuda et al., Long-lived neutral-kaon flux measurement for the KOTO experiment, PTEP 2016 (2016) 013 C03 [physics.ins-det/1509.03386]

[31] J. Comfort et al., Proposal for $K_{L} \rightarrow \pi^{0} v \bar{v}$ Experiment at J-Parc, http: //koto.kek.jp/pub/p14.pdf (2006) 